\title{
PTPRB promotes metastasis of colorectal carcinoma via inducing epithelial- mesenchymal transition
}

Xingyue Weng ${ }^{1}$, Wei Chen², Wangxiong Hu', Kailun Xu', Lina Qi', Jiani Chen', Demin Lu', Yinkuan Shao ', Xi Zheng $\mathbb{D}^{1}$, Chenyang $\mathrm{Ye}^{1}$ and Shu Zheng ${ }^{1}$

\begin{abstract}
Dysregulation of protein tyrosine phosphatase, receptor type B (PTPRB) correlates with the development of a variety of tumors. Here we show that PTPRB promotes metastasis of colorectal cancer (CRC) cells via inducing epithelialmesenchymal transition (EMT). We find that PTPRB is expressed at significantly higher levels in CRC tissues compared to adjacent nontumor tissues and in CRC cell lines with high invasion. PTPRB knockdown decreased the number of invasive CRC cells in an in vitro wound healing model, and also reduced tumor metastasis in vivo. Conversely, PTPRB overexpression promoted CRC cell invasion in vitro and metastasis in vivo. PTPRB overexpression decreased vimentin expression and promoted E-cadherin expression, consistent with promotion of EMT, while PTPRB knockdown had the opposite effect. Hypoxic conditions induced EMT and promoted invasion in CRC cells, but these effects were eliminated by PTPRB knockdown. EMT blockade via TWIST1 knockdown inhibited the migration and invasiveness of CRC cells, and even increased PTPRB expression could not reverse this effect. Altogether, these data support the conclusion that PTPRB promotes invasion and metastasis of CRC cells via inducing EMT, and that PTPRB would be a novel therapeutic target for the treatment of CRC.
\end{abstract}

\section{Introduction}

Colorectal cancer (CRC) is one of the most common cancers, and is diagnosed in more than 1 million patients each year ${ }^{1}$. Moreover, CRC is the fourth most common cause of cancer-related deaths after lung, liver, and stomach cancer ${ }^{2}$. Surgical resection has been considered as the most effective treatment for patients with CRC. However, despite significant advances in perioperative management and expansion of screening programs, CRC mortality continues to increase rapidly worldwide ${ }^{3}$.

\footnotetext{
Correspondence: Shu Zheng (zhengshu@zju.edu.cn)

${ }^{1}$ Cancer Institute (Key Laboratory of Cancer Prevention and Intervention, China National Ministry of Education), The Second Affiliated Hospital, Zhejiang University School of Medicine, Hangzhou, Zhejiang 310009, China

${ }^{2}$ Cancer Institute of Integrated Traditional Chinese and Western Medicine, Zhejiang Academy of Traditional Chinese Medicine, Tongde Hospital of

Zhejiang Province, Hangzhou, Zhejiang 310012, China

These author contributed equally: Xingyue Weng, Wei Chen

Edited by A. Stephanou
}

Metastasis and recurrence are believed to be responsible for limiting long-term survival of patients with CRC, and there is an estimated recurrence rate of $29-63 \%$ among patients with stage II-III CRC ${ }^{4}$. According to previously reported data, the long-term survival of CRC patients with liver metastasis is rarely longer than three years ${ }^{5}$. Therefore, further understanding the vital mechanisms underlying CRC progression is urgent for developing new therapeutic strategies to improve prognosis.

Protein tyrosine phosphatase, receptor type B (PTPRB), also known as VE-PTP and RPTP $\beta$, is located on chromosome $12 \mathrm{q} 15^{6}$. The chromosome 12q15 locus contains multiple proliferation-related genes, and patients with deletions of the chromosome $12 \mathrm{q} 15$ region commonly present with global developmental delay and growth retardation ${ }^{7}$. PTPRB belongs to the protein tyrosine phosphatase family, and consists of an extracellular domain with multiple fibronectin type III-like domains, a

\section{(c) The Author(s) 2019}

(c) (i) Open Access This article is licensed under a Creative Commons Attribution 4.0 International License, which permits use, sharing, adaptation, distribution and reproduction c. in any medium or format, as long as you give appropriate credit to the original author(s) and the source, provide a link to the Creative Commons license, and indicate if changes were made. The images or other third party material in this article are included in the article's Creative Commons license, unless indicated otherwise in a credit line to the material. If material is not included in the article's Creative Commons license and your intended use is not permitted by statutory regulation or exceeds the permitted use, you will need to obtain permission directly from the copyright holder. To view a copy of this license, visit http://creativecommons.org/licenses/by/4.0/. 
single intracellular catalytic domain with C-terminal phosphorylation sites, and a transmembrane domain. Multiple studies have previously demonstrated that PTPRB plays critical roles in regulating various biological processes depending on binding and dephosphorylation of many types of receptor tyrosine kinases $(\mathrm{RKTs})^{8}$. Soady et al. showed that PTPRB negatively regulates branching morphogenesis in the mammary epithelium, dependent on inhibition of FGFR activation and ERK1/2 phosphorylation ${ }^{9}$. Genetic studies in both mammals and invertebrate model systems have shown that the RPTP family is essential for tubular organ development ${ }^{10}$.

Moreover, dysregulation of PTPRB function and expression has been shown to correlate with carcinogenesis and tumor progression in multiple cancer types ${ }^{11-13}$. Qi et al. reported that PTPRB could decrease the level of Src phosphorylation, resulting in reduced cell proliferation and inhibitory tumorigenesis in non-small cell lung carcinoma ${ }^{14}$. Activation of the insulin signaling pathway is a very common phenomenon in many cancers ${ }^{15}$. PTPRB preferentially dephosphorylates the insulin receptor at Y960 and Y1146, suppressing insulin-induced activation of the insulin receptor and $\mathrm{Akt}^{16}$.

In this study, we used CRC cell lines to explore the expression of PTPRB and to analyze the biological function of PTPRB protein, with a focus on invasion and epithelialmesenchymal transition (EMT). The results confirmed that PTPRB is highly expressed in CRC tissues and CRC cell lines with high invasion, and that PTPRB knockdown suppresses CRC cell migration and invasion in vitro and metastasis in vivo dependent on inhibition of EMT.

\section{Results}

PTPRB is associated with the motility and invasiveness of CRC cells

The motility and invasiveness of three CRC cell lines (LOVO, HCT116, and HT29) were analyzed by the transwell assay and wound healing assay. After $36 \mathrm{~h}$ incubation, the wound distance was reduced by $70 \%$ in LOVO, 50\% in HCT116, and 30\% in HT29 cells (Fig. 1a). Similarly, the frequency of invasiveness was the highest in LOVO, intermediate in HCT116, and lowest in HT29 cells (Fig. 1b). Interestingly, the expression of PTPRB at both the transcriptional level (Fig. 1c) and protein level (Fig. 1d) was high in LOVO, intermediate in HCT116, and low in HT29 cells. These results indicate that the expression of PTPRB in cells with different motility rates was different. The expression of PTPRB in cells with high motility rate was higher.

PTPRB promotes migration and invasion in CRC cell lines siRNAs targeting PTPRB and PTPRB overexpression plasmids were, respectively, used to decrease and upregulate PTPRB expression in three CRC cell lines. Western blotting showed the transfection efficiency of PTPRB siRNA or PTPRB plasmid in all cell lines (Fig. 2a). The PTPRB-siRNA-transfected CRC cells showed a significant decrease in motility compared to the negative siRNA. Conversely, PTPRB overexpression accelerated the rate of wound healing in the CRC cell lines (Fig. 2b). A similar effect of PTPRB on invasiveness was observed. PTPRB knockdown significantly decreased the number of invasive cells compared to negative siRNA, while PTPRB overexpression promoted invasiveness (Fig. 2c).

\section{PTPRB regulates EMT in CRC cell lines}

The expression level of vimentin ranged from high to low in LOVO, HCT116, and HT29 cells, and an opposite trend in expression was observed for E-cadherin (Fig. 3a, b). This result suggested that EMT was essential for CRC cell migration and invasion. We therefore investigated the relationship between PTPRB expression and the EMT process in CRC cells. After decreasing PTPRB expression, CRC cells showed reduced vimentin expression and increased E-cadherin expression. On the contrary, PTPRB overexpression promoted vimentin expression and downregulated E-cadherin expression (Fig. 3c). In line with the results of western bolt, PTPRB siRNA could decrease the fluorescence of vimentin and increased the fluorescence of E-cadherin, while PTPRB overexpression had the opposite effect (Fig. 3d). At the same time, PTPRB siRNA could reduce twist expression while PTPRB overexpression increased twist expression (Fig. S). Finally, a siRNA-targeting Twist1 was transfected into CRC cells to inhibit the EMT process. As anticipated, Twist1 knockdown significantly inhibited CRC cell migration and invasion (Fig. 4a, b). In addition, Twist1 knockdown also eliminated the effect of PTPRB overexpression on promoting CRC cells invasion.

\section{PTPRB knockdown inhibits hypoxia-induced metastasis in CRC cells}

Hypoxia has a significant role in inducing EMT and metastasis in multiple cancers including $\mathrm{CRC}^{17}$. Hypoxiainducible factor (HIF) is the key factor of physiological and pathological hypoxia response, which is significantly expressed in hypoxic environment. The qRT-PCR assay showed the level of HIF-1 $\alpha$ was increased under hypoxia compared to the normoxia condition, indicating hypoxic condition was generated (Fig. 5a). After culture for $36 \mathrm{~h}$ in hypoxic conditions, CRC cells showed increased migration and invasion compared to control groups (Fig. 5b, c). However, PTPRB knockdown could reverse the effect of hypoxia on promoting migration and invasion. In addition, we had detected the effect of PTPRB on Twist under hypoxia. The twist expression was increased under hypoxia while PTPRB siRNA could reverse the effect of hypoxia on twist (Fig. S). 

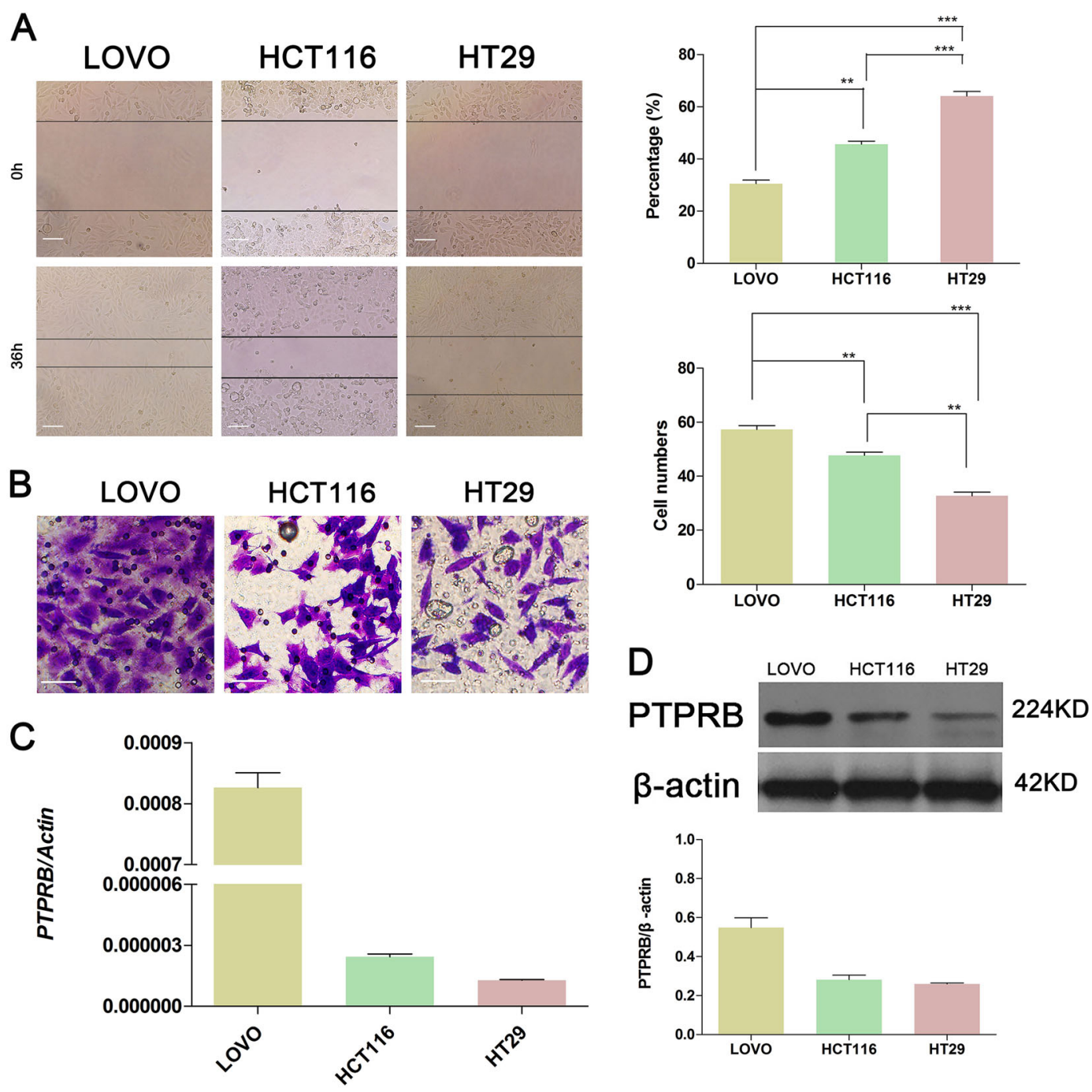

Fig. 1 a The wound recovery ratio after incubation for $36 \mathrm{~h}$ for LOVO, HCT116, and HT29 cells, ${ }^{* *} P<0.01,{ }^{* *} P<0.001$. $\mathbf{b}$ The number of invasive cells in LOVO, HCT116, and HT29 lines, ${ }^{* *} P<0.01$, ***P $<0.001$. c, d The expression level of PTPRB in LOVO, HCT116, and HT29 cells was detected at the transcript level by RT-PCR and protein level by western blotting analysis

\section{PTPRB promotes tumor metastasis in vivo}

To determine the effects of PTPRB expression level on tumor metastasis in vivo, PTPRB shRNA and PTPRB plasmids were transfected into CRC cells. Western blotting showed the transfection efficiency of PTPRB shRNA or PTPRB plasmid in HCT116 cells (Fig. 6a). Compared to mice injected with control cells, PTPRB knockdown cells inhibited tumor metastasis in the whole body (Fig. 6b), such as lung (Fig. 6c), while PTPRB overexpression increased tumor metastasis. Moreover, western blot analysis (Fig. 6d) and RT-PCR (Fig. 6e) revealed that PTPRB knockdown decreased vimentin expression and increased E-cadherin expression in tumor tissues. PTPRB overexpression showed an opposite effect on vimentin and Ecadherin expression.

\section{PTPRB is highly expressed in CRC}

To analyze the expression of PTPRB in CRC, tumor tissues and adjacent nontumor tissues from 100 patients were assayed by immunohistochemistry and RT-PCR. As seen in Fig. $7 \mathrm{a}-\mathrm{c}$, the level of PTPRB mRNA and protein was significantly higher in CRC than in adjacent tissues. But as shown as in Fig. 7d, survival analysis indicated that there was no significantly difference between low expression and high expression of PTPRB.

\section{Discussion}

PTPRB has been investigated in multiple primary malignancies and tumor cell lines ${ }^{14,18,19}$. However, there are few studies on the relationship between PTPRB function and CRC. This study firstly demonstrated that 


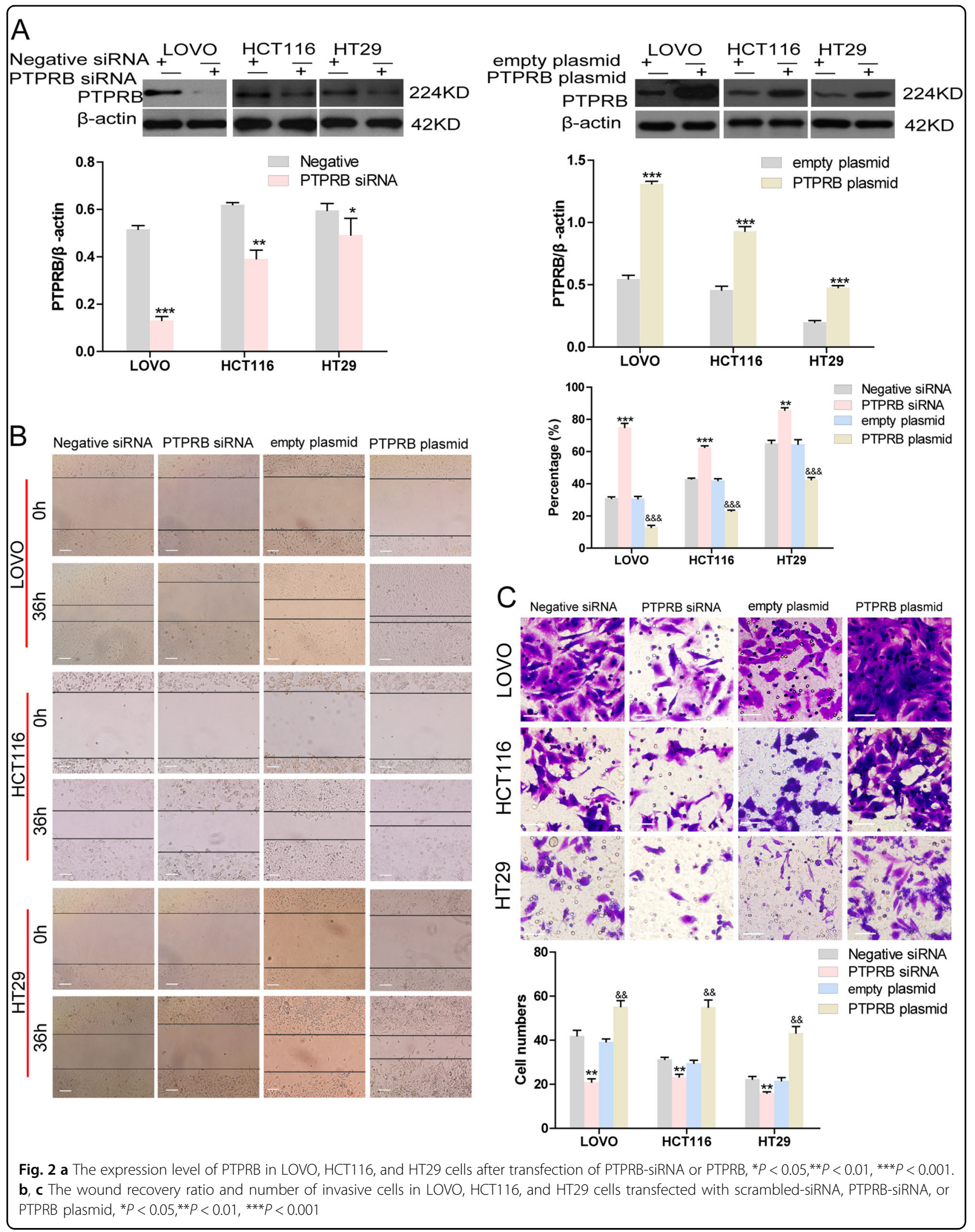




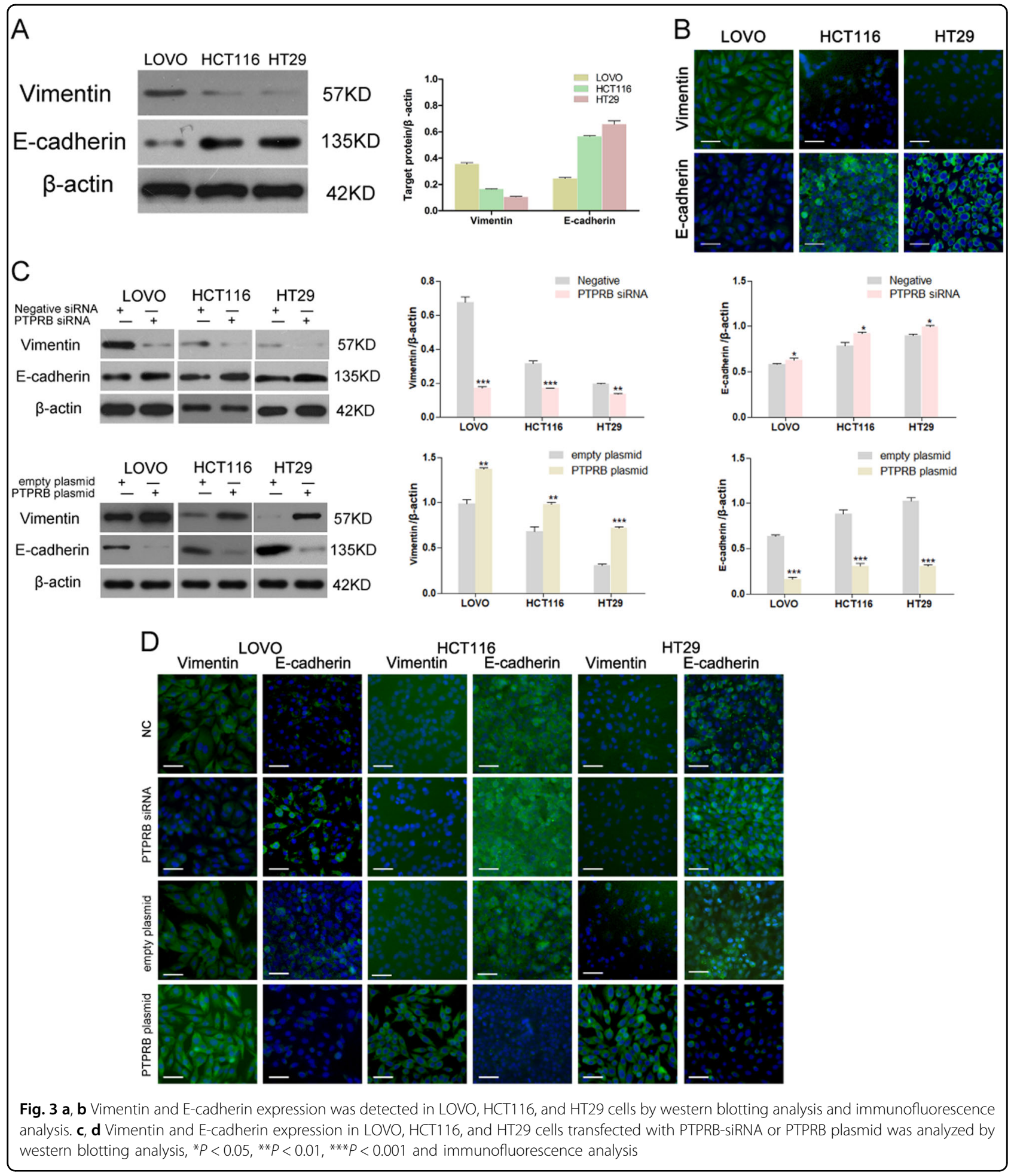

PTPRB is highly expressed in CRC tissues compared with adjacent nontumor tissues and in CRC cells with high metastasis potential. Metastasis is well known to be the main cause of CRC-related death, and more than $50 \%$ of patients with $\mathrm{CRC}$ will present with liver metastases during their lifespan ${ }^{20}$. PTPRB-siRNA significantly suppressed migration and invasion of CRC cells in vitro and inhibitory tumorigenesis in vivo. This result suggested that PTPRB was a potential molecular target against CRC metastasis. PTPRB could catalyze the dephosphorylation 


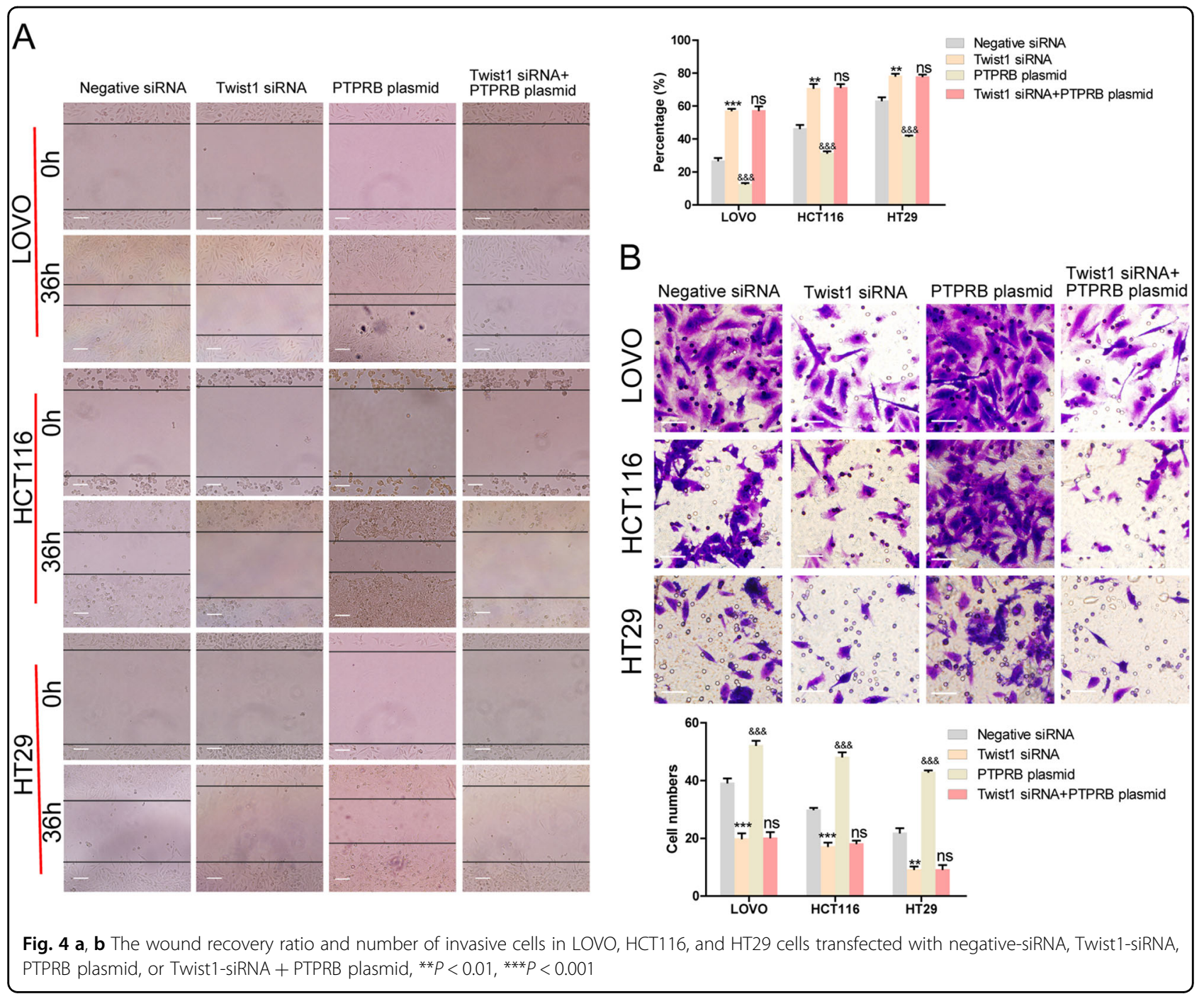

of phosphotyrosine residues, and along with protein tyrosine kinases could modulate the levels of phosphotyrosine modification in tumor cells.

Reversible phosphorylation of tyrosine accounts for less than $1 \%$ of the phosphoproteome, but it plays a disproportionately significant role in many diseases including cancer initiation and development ${ }^{21}$. Nearly $50 \%$ of the 90 human tyrosine kinases are implicated in cancer ${ }^{22}$, and many different RTKs are direct targets for protein tyrosine phosphatases ${ }^{23-25}$. Hence, PTPRB could regulate the proliferation, migration, invasion, and tumorigenesis of tumor cells as an essential regulator of the RTK signaling network.

Understanding the molecular mechanisms underlying how CRC cells acquire invasive and metastatic properties is essential for the development of effective strategies for CRC therapy. Increasing evidence supports the view that EMT could endow tumor cells with metastatic features via inducing loss of epithelial characteristics, for instance, cell depolarization, cell-cell disconnection, and transformation into an elongated, fibroblast-like morphol$\mathrm{ogy}^{26}$. EMT is characterized by decreased expression of epithelial cell junction proteins such as E-cadherin, occludins, and claudins, and the upregulation of mesenchymal adhesion genes encoding, for instance, vimentin, fibronectin, and $\mathrm{N}$-cadherin ${ }^{27}$. Among these, downregulation of E-cadherin expression results in the destabilization of adherens junctions and is a primary step for cancer metastasis. Therefore, we investigated the relationship between PTPRB and E-cadherin expression in CRC cells. Western blot analysis and immunofluorescence analysis showed that PTPRB overexpression induced higher vimentin expression and lower E-cadherin protein expression, while PTPRB knockdown resulted in an opposite effect on vimentin and E-cadherin protein expression in CRC cells. These results suggested that PTPRB plays a significant role in regulating EMT. 


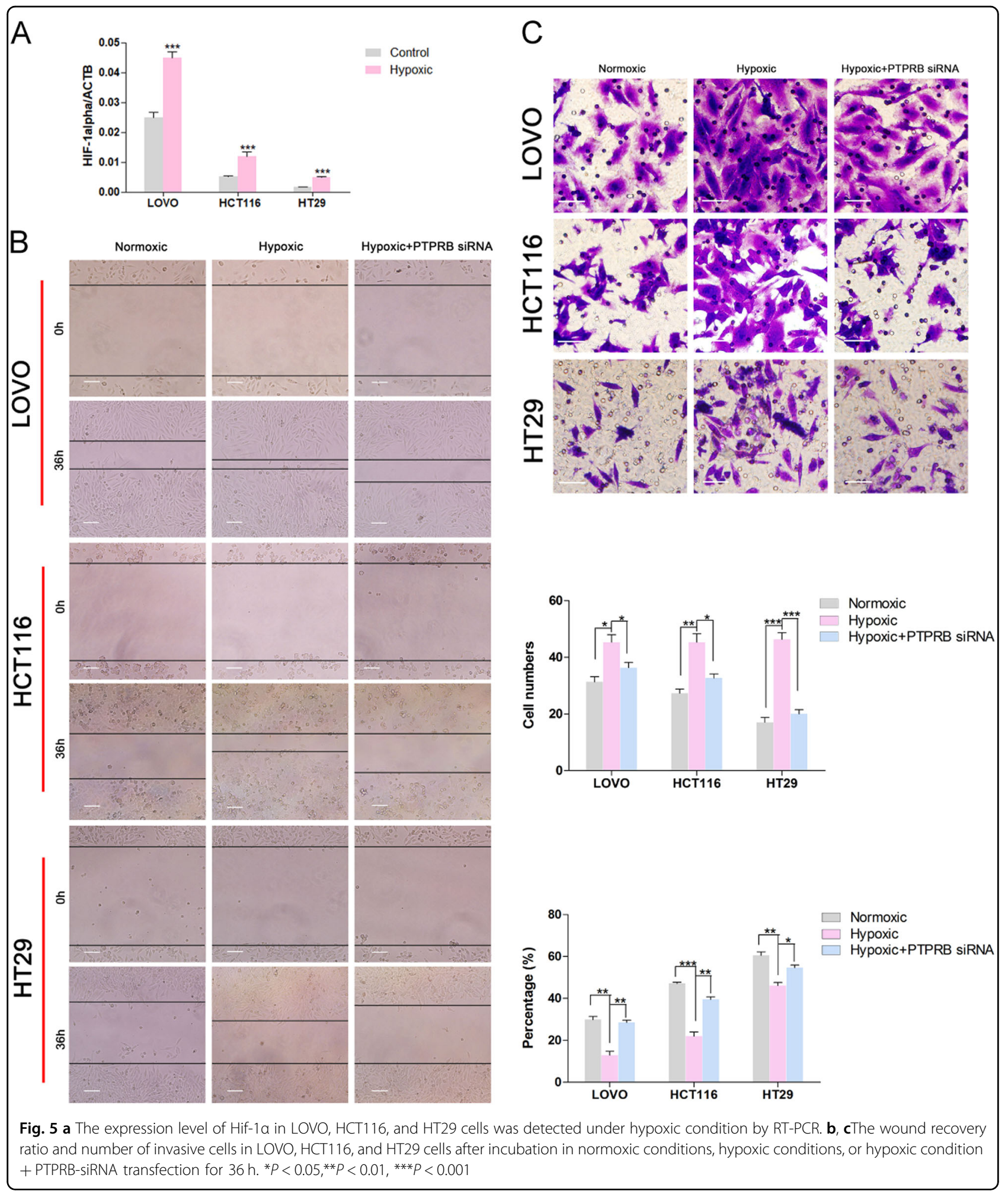

The regulation of epithelial and mesenchymal markers requires robust transcriptional machinery, consisting of three major groups of transcription factors: the TWIST family of basic helix-loop-helix transcription factors
(TWIST1/TWIST2) $^{28}$, the zinc finger E-box binding homeobox (ZEB) family of transcription factors (ZEB1/ ZEB2 ${ }^{29}$, and the SNAIL family of zinc-finger transcription factors (SNAIL/SLUG) ${ }^{30}$. In this study, Twist1 siRNA 


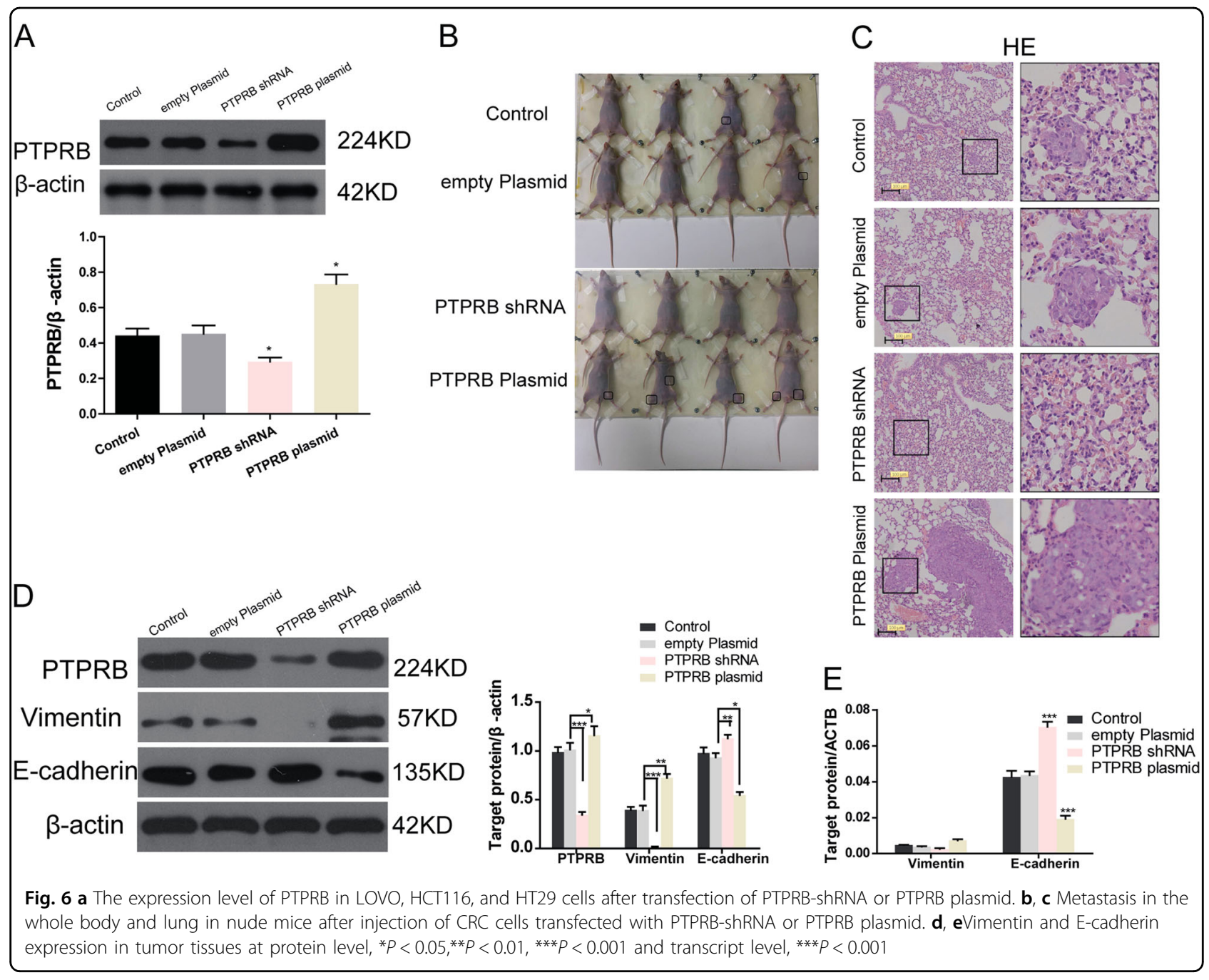

was transfected into CRC cells to inhibit the EMT process. The results showed that Twist1 knockdown could eliminate the enhanced capacity for migration and invasion induced by PTPRB overexpression, suggesting that EMT may be the mechanism underlying the capacity for PTPRB to promote invasion.

In cancer cells, EMT is abnormally modulated by extracellular stimuli derived from the tumor microenvironment, for instance, inflammatory cytokines, growth factors, as well as intratumoral physical stresses such as hypoxia ${ }^{24}$. Therefore, we further cultured CRC cells in hypoxic conditions to induce EMT. We found that PTPRB knockdown inhibited expression of EMT markers even under hypoxic conditions. Altogether, these results provide compelling evidence supporting the prometastatic and pro-EMT function of PTPRB in CRC.

The in vitro experiments showed an antimetastasis role of PTPRB knockdown in CRC, and thus prompted us to evaluate its functions in tumor metastasis in vivo. The results showed that stable knockdown of PTPRB led to a significant inhibition of metastasis in lung and rectocolon in nude mice. Moreover, tumor sections from PTPRB knockdown xenografts showed lower levels of vimentin expression and upregulation of E-cadherin expression. Finally, the RT-PCR assay revealed that PTPRB was significantly upregulated in CRC tissues compared to adjacent nontumor tissues. Consistent with mRNA expression, immunohistochemical analysis showed that PTPRB was highly expressed in tumor tissues at the protein level. But, survival analysis showed that there was no significantly difference between low expression and high expression of PTPRB. It may be that the number of cases is not enough, or the meaning of cytoplasmic nuclear protein expression may be different. Anyway, these findings provide more comprehensive insight into the value of PTPRB as a potential CRC therapy target.

In conclusion, our study shows that PTPRB is upregulated in CRC. Functional data from both in vivo and in vitro assays strongly support the inference that PTPRB overexpression promotes the metastasis of CRC. 

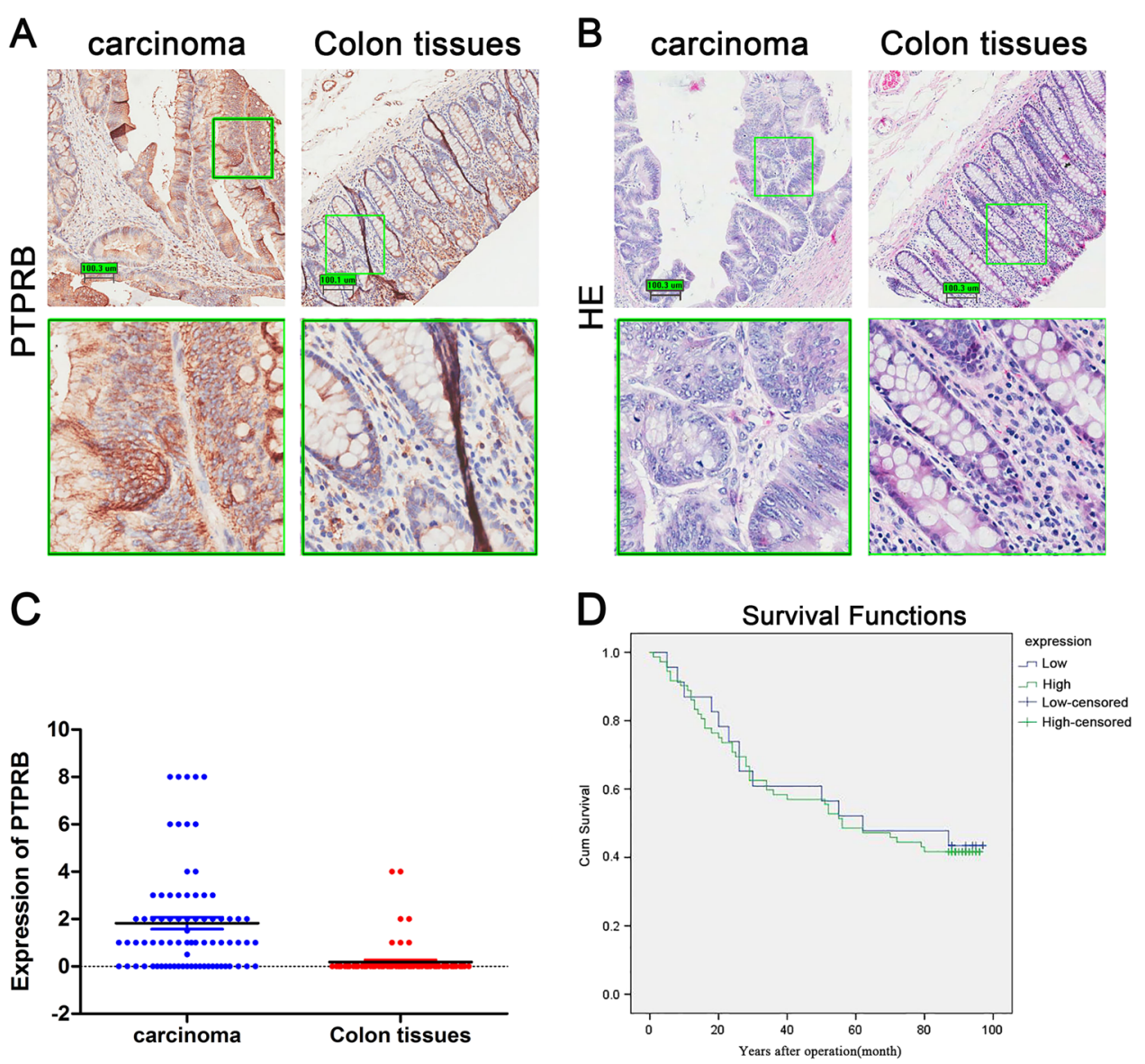

Fig. 7 aThe level of PTPRB expression in CRC and adjacent tissues was observed by histochemical stain. $\mathbf{b}$ HE staining of CRC tissues and adjacent nontumor tissues. c The expression level of PTPRB mRNA in CRC and adjacent tissues. $\mathbf{d}$ This study was used Kaplan-Meier survival analysis and logrank statistical test to analyze the survival of PTPRB in 80 cancer tissues by single factor analysis

Moreover, our study revealed the effect of PTPRB on promoting EMT, as reflected in increased vimentin and decreased E-cadherin expression potential, which provides a potential comprehensive mechanism by which PTPRB may promote metastasis.

\section{Materials and methods}

\section{Clinical specimen collection and cell culture}

Samples from 100 patients undergoing CRC resection were collected from Shanghai Outdo Biotech Company. Informed consent was obtained from all patients, and the experimental protocols agreed with local ethics committee regulations. Three CRC cell lines (LOVO, HCT116, and HT29) were purchased from Cell Bank of Type Culture Collection of Chinese Academy of Sciences, Shanghai Institute of Cell Biology, Chinese Academy of Sciences. LOVO and HT29 cells were cultured in 1640 complete medium supplemented with $10 \%$ fetal bovine serum (FBS) while HCT116 cells were cultured in McCoy's 5A (Modified) Medium with $10 \% \mathrm{FBS}$ at $37^{\circ} \mathrm{C}$ in a humidified incubator with $5 \% \mathrm{CO}_{2}$. Furthermore, the hypoxic condition in a hyposic incubator is $5 \% \mathrm{CO}_{2}, 94 \%$ $\mathrm{N}_{2}$, and $1 \% \mathrm{O}_{2}$ at $37^{\circ} \mathrm{C}$.

\section{Transfection assay}

The cells were seeded in a six-well plate at $1 \times 105$. When $80 \%$ confluence of cells, the siRNA (final concentration $100 \mathrm{nM}$, Ribobio, Guangzhou, China) or plasmid ( $2 \mu \mathrm{g}$, Hanbio, Shanghai, China) was transfected into the cells with liposome lipo 2000 (Invitrogen, California, USA). The lipo was diluted $(5 \mu \mathrm{l} /$ well $)$ and the siRNA or plasmid was diluted at the calculated concentration by OPTIM-MEM (Gibco Company, Massachusetts, USA), respectively, and then the two were mixed in a proportional manner for $15 \mathrm{~min}$. The cells were transfected by the mixed medium in an incubator for $6-8 \mathrm{~h}$ and then cultured in a normal culture medium. For in vivo study, the siRNA proved effective in vitro was packaged into lentivirus (Hanbio, Shanghai, China). In order to generate stable transfected cell lines, puromycin and G418 were 
separately used to screen for lentivirus and plasmid transfected cells. The survival cells were collected and cultured. Western Blot was used to detect the transfection efficiency. The siRNA sequences targeting PTPRB and Twist 1 are listed as follows:

Twist 1 ,

Twist1-homo-1575 5' GGUGUCUAAAUGCAUUCAU TT $3^{\prime}$

\section{5' AUGAAUGCAUUUAGACACCTT 3'}

Twist1-homo-810 5' GGUACAUCGACUUCCUCUA TT $3^{\prime}$

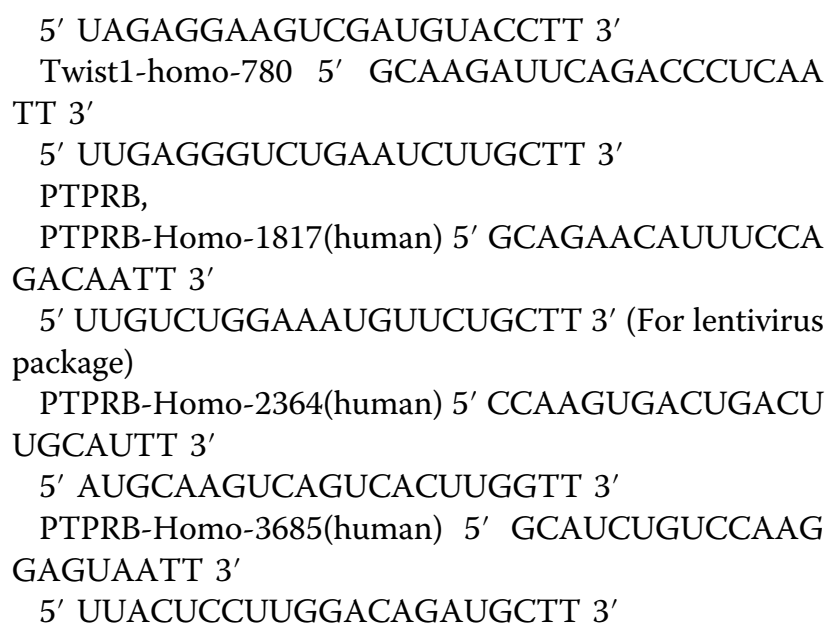

\section{Total RNA extraction and RT-PCR}

Total RNA from cells and CRC tissues were extracted using a TRIzol Total RNA extraction Kit (Invitrogen Co.), and reverse-transcribed to cDNA using a TaqMan Reverse Transcription Kit (Applied Biosystems). Quantitative real-time PCR (qRT-PCR) analysis was performed using a Takara SYBR Premix Ex Taq system (Applied Biosystems). All experiments were performed in triplicate. The nucleotide sequences of the primers used for qRTPCR (Shanghai Sangon Biological Engineering Technology Services Co., Ltd) are as follows:

PTPRB,

Ptprb-F 5' CACAGAGATGCAATCTACTCGAGAC 3' Ptprb-R 5' CAACAGAAATGGCTGGCACC 3'

Actin,

Actin-F 5' TGGCACCCAGCACAATGAA 3' Actin-R 5' CTAAGTCATAGTCCGCCTAGAAGCA 3' Vimentin,

VIM-F 5' TGAGTACCGGAGACAGGTGCAG 3' VIM-R $5^{\prime}$ TAGCAGCTTCAACGGCAAAGTTC $3^{\prime}$ E-cadherin,

E-cadherin-F 5' TACACTGCCCAGGAGCCAGA $3^{\prime}$

E-cadherin-R 5' TGGCACCAGTGTCCGGATTA 3'

\section{Western blotting analysis}

Protein from cells and tissues were lysed using RIPA buffer (Beyotime, Jiangsu, China) supplemented with protease inhibitors PMSF (Beyotime). Total protein concentration was determined by the $\mathrm{BCA}$ assay. In all, 20-50 $\mu \mathrm{g}$ total protein was subjected to electrophoretic separation in SDS-PAGE gel analysis, and then transferred to PVDF membranes at $350 \mathrm{~mA}$ for $90 \mathrm{~min}$. Subsequently, the membranes were blocked using $5 \%$ non-fat milk in TBS supplemented with $0.1 \%$ Tween- 20 for $1-2 \mathrm{~h}$. After washing three times, the membranes were incubated with primary antibodies overnight at $4{ }^{\circ} \mathrm{C}$, and further incubated with an appropriate secondary antibody for $2 \mathrm{~h}$. The expression levels of target proteins were visualized using enhanced chemiluminescence.

Primary antibodies against PTPRB (1:1000), Vimentin (1:1000), E-cadherin (1:1000), Goat antiRabbit HRP antibody, and Goat antiMouse HRP antibody were purchased from Cell Signaling Technology (Beverly, MA, USA).

\section{Tissue microarray and Immunohistochemistry}

The expression level of PTPRB in tissue was determined by tissue microarray (TMA). TMAs were assembled by manual tissue punch. Different samples were drilled from selected tissue areas and assembled into new paraffin blocks. The histological cores are $2 \mathrm{~mm}$ in diameter and $4-6 \mathrm{~mm}$ in length. The immunohistochemistry stainings were performed on $4 \mu \mathrm{m}$ sections. The sections were deparaffinized and rehydrated, then subjected to heatinduced epitope retrieval. The activity of endogenous peroxidase was quenched with $3 \%$ hydrogen peroxide, and the sections were then blocked using 5\% FBS in TBST. After washing three times, the primary monoclonal antibody was added and sections were incubated overnight at $4{ }^{\circ} \mathrm{C}$. After incubation with HRP-conjugated secondary antibodies for $1 \mathrm{~h}$ at room temperature, protein expression was visualized using 3,3'-diaminobenzidine with hematoxylin stain for contrast.

the standardization of the original experimental data:

1. Staining intensity score: 0 points (negative), 1 point $(1+), 2$ points $(2+), 3$ points $(3+)$;

2. Staining positive rate score: 0 points (negative), 1 point (1-25\%), 2 points ( $26-50 \%), 3$ points (51-75\%), 4 points (76-100\%);

3. Total score and grouping: The product of "staining intensity score" and "staining positive rate score" is grouped into total scores; $\leq 2$ is divided into antibody low expression group, $>2$ is divided into antibody high expression group. (depending on the distribution of data).

\section{Wound healing assay}

A total of $3 \times 10^{5}$ CRC cells were seeded in six-well plates containing $2 \mathrm{ml}$ culture medium. After achieving 80-90\% confluence, an open wound was scratched into the cell monolayer using $200 \mu \mathrm{l}$ pipettes and the medium was replaced with serum-free medium. The capacity of 
cell migration was determined as the percentage of recovery following a $36 \mathrm{~h}$ incubation period.

\section{Transwell invasion experiment}

A total of $1 \times 105$ CRC cells in $200 \mu \mathrm{l}$ serum-free 1640 medium were distributed into the upper chambers of each well (24-well insert; 8-mm pore size; Millipore, Billerica, MA, USA) coated with Matrigel (BD Bioscience). Culture medium supplemented with $10 \%$ FBS was added in the lower chambers as a chemoattractant. After incubation for $24 \mathrm{~h}$, cells remaining in the upper chambers were wiped off, and the cells on the reverse aspect of the Matrigel membrane were fixed with 4\% paraformaldehyde for $20 \mathrm{~min}$. The number of invasive cells was recorded after staining with $0.4 \%$ crystal violet.

\section{Animal studies}

BALB/c-nu male nude mice (age: 4-5 weeks; weight: 20-25 g) (Experimental Animal Center of Zhejiang University, Hangzhou, Zhejiang, China) were grouped into Control, empty plasmid, shRNA-PTPRB (lentivirus), and PTPRB plasmid groups, with at least eight mice in each group and approved by the Second Affiliated Zhejiang Hospital, Zhejiang University of Medical Ethics Committee and the Medical Faculty Ethics Committee of the Second Affiliated Zhejiang Hospital, Zhejiang University. All experiments were performed in accordance with the Guide for the Care and Use of Experimental Animals guidelines and regulations. HCT116 cells or stable tranfected cells at the logarithmic phase of growth were collected, counted and suspected by physiological saline solution. Nude mice were injected with $0.2 \mathrm{ml}$ cell suspension $\left(1.0 \times 10^{7}\right.$ cells $\left./ \mathrm{ml}\right)$ via tail vein. Six weeks later, took photograph. Next, lung tissue was isolated, fixed in $10 \%$ formalin for HE staining.

\section{Statistical analysis}

All experimental data are presented as mean with SD. The difference between two groups was analyzed using unpaired Student's $t$-test. Survival analysis curves were plotted using the Kaplan-Meier method. $P<0.05$ was considered to be statistically significant. Statistical analysis was performed using SPSS 17.0 software (SPSS).

\footnotetext{
Acknowledgments

This study was supported by Key Projects in the National Science \& Technology Pillar Program during the Twelfth Five-year Plan Period (2014BAI09B07); the National High Technology Research and Development Program of China (863 Program) (2012AA02A506); the National Science and Technology Major Project (2011ZX09307-001-05); the National High Technology Research and Development Program of China (863 Program) (2012AA02A204) and Training Program of the Major Research Plan of the National Natural Science Foundation of China (91229104).
}

\section{Publisher's note}

Springer Nature remains neutral with regard to jurisdictional claims in published maps and institutional affiliations.

Supplementary Information accompanies this paper at (https://doi.org/ 10.1038/s41419-019-1554-9).

Received: 30 October 2018 Revised: 20 March 2019 Accepted: 26 March 2019

Published online: 30 April 2019

\section{References}

1. Siegel, R. L., Miller, K. D. \& Jemal, A. Cancer statistics, 2016. CA Cancer J Clin 66, 7-30 (2016).

2. Siegel, R., Desantis, C. \& Jemal, A. Colorectal cancer statistics, 2014. CA Cancer J Clin 64, 104-117 (2014).

3. Brenner, H., Kloor, M. \& Pox, C. P. Colorectal cancer. Lancet 383, 1490-1502 (2014).

4. Manfredi, S. et al. Incidence and patterns of recurrence after resection for cure of colonic cancer in a well defined population. Br. J. Surg. 93, 1115-1122 (2006).

5. Cunningham, D. et al. Cetuximab monotherapy and cetuximab plus irinotecan in irinotecan-refractory metastatic colorectal cancer. N. Engl. J. Med. 351, 337-345 (2004)

6. Harder, K. W., Anderson, L. L., Duncan, A. M. \& Jirik, F. R. The gene for receptorlike protein tyrosine phosphatase (PTPRB) is assigned to chromosome 12q15>q21. Cytogenet. Cell Genet. 61, 269-270 (1992).

7. Alesi, V. et al. Reassessment of the $12 q 15$ deletion syndrome critical region. Eur. J. Med. Genet. 60, 220-223 (2017).

8. Barr, A. J. et al. Large-scale structural analysis of the classical human protein tyrosine phosphatome. Cell 136, 352-363 (2009).

9. Soady, K. J. et al. The receptor protein tyrosine phosphatase PTPRB negatively regulates FGF2-dependent branching morphogenesis. Development $\mathbf{1 4 4}$ 3777-3788 (2017).

10. Jeon, M. \& Zinn, K. R3 receptor tyrosine phosphatases: conserved regulators of receptor tyrosine kinase signaling and tubular organ development. Semin. Cell Dev. Biol. 37, 119-126 (2015).

11. Sena, J. A., Wang, L. \& Hu, C. J. BRG1 and BRM chromatin-remodeling complexes regulate the hypoxia response by acting as coactivators for a subset of hypoxia-inducible transcription factor target genes. Mol. Cell. Biol. 33, 3849-3863 (2013)

12. Ciribilli, Y. \& Borlak, J. Oncogenomics of c-Myc transgenic mice reveal novel regulators of extracellular signaling, angiogenesis and invasion with clinical significance for human lung adenocarcinoma. Oncotarget 8, 101808-101831 (2017).

13. Ding, L. et al. Clonal architectures and driver mutations in metastatic melanomas. Plos One 9, e111153 (2014).

14. Qi, Y., Dai, Y. \& Gui, S. Protein tyrosine phosphatase PTPRB regulates Src phosphorylation and tumour progression in NSCLC. Clin. Exp. Pharmacol. Physiol. 43, 1004-1012 (2016).

15. Mutgan, A. C. et al. Insulin/IGF-driven cancer cell-stroma crosstalk as a novel therapeutic target in pancreatic cancer. Mol. Cancer 17, 66 (2018).

16. Shintani, T. et al. The R3 receptor-like protein tyrosine phosphatase subfamily inhibits insulin signalling by dephosphorylating the insulin receptor at specific sites. J. Biochem. 158, 235-243 (2015).

17. Tsai, Y. P. et al. TET1 regulates hypoxia-induced epithelial-mesenchymal transition by acting as a co-activator. Genome Biol. 15, 513 (2014).

18. Aso, $Y$. et al. Induction of genes expressed in endothelial cells of the corpus callosum in the chronic cerebral hypoperfusion rat model. Pathobiology $\mathbf{8 4}$, 25-37 (2017).

19. Chen, $\mathrm{H}$. et al. A seven-gene signature predicts overall survival of patients with colorectal cancer. Oncotarget 8, 95054-95065 (2017).

20. Misiakos, E. P., Karidis, N. P. \& Kouraklis, G. Current treatment for colorectal liver metastases. World J. Gastroenterol. 17, 4067-4075 (2011).

21. Ptacek, J. \& Snyder, M. Charging it up: global analysis of protein phosphorylation. Trends Genet. 22, 545-554 (2006).

22. Del Rosario, A. M. \& White, F. M. Quantifying oncogenic phosphotyrosine signaling networks through systems biology. Curr. Opin. Genet. Dev. 20, 23-30 (2010). 
23. Behjati, S. et al. Recurrent PTPRB and PLCG1 mutations in angiosarcoma. Nat. Genet. 46, 376-379 (2014).

24. Zhang, J. et al. Hypoxia-inducible factor-1alpha/interleukin-1beta signaling enhances hepatoma epithelial-mesenchymal transition through macrophages in a hypoxic-inflammatory microenvironment. Hepatology. https://oi.org/ 10.1002/hep.29681 (2017).

25. Koch, S. et al. In situ proximity ligation assay (in situ PLA) to assess PTP-protein interactions. Methods Mol Biol 1447, 217-242 (2016).

26. Vu, T. \& Datta, P. K. Regulation of EMT in colorectal cancer: a culprit in metastasis. Cancers. 9, https://doi.org/10.3390/cancers9120171 (2017).
27. Vinson, K. E., George, D. C., Fender, A. W., Bertrand, F. E. \& Sigounas, G. The Notch pathway in colorectal cancer. Int. J. Cancer 138, 1835-1842 (2016).

28. Jung, H. Y. \& Yang, J. Unraveling the TWIST between EMT and cancer stemness. Cell Stem Cell 16, 1-2 (2015).

29. Krebs, A. M. et al. The EMT-activator Zeb1 is a key factor for cell plasticity and promotes metastasis in pancreatic cancer. Nat. Cell Biol. 19, 518-529 (2017).

30. Cano, A. et al. The transcription factor snail controls epithelialmesenchymal transitions by repressing E-cadherin expression. Nat. Cell Biol. 2, 76-83 (2000). 